RESEARCH HIGHLIGHTS
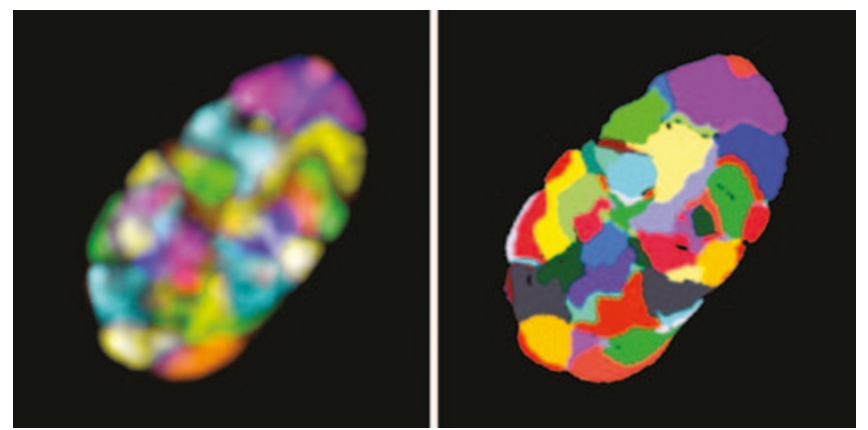

Image courtesy of Andreas Bolzer and Thomas Cremer, Ludwig Maximilians University, Munich

\title{
Exploring chromatin architecture by FISHing in 3D
}

Gene expression is regulated at many levels, including the DNA-sequence, chromatin and nuclear levels. Perhaps the area about which least is known is how nuclear organization influences gene expression. Does the level of expression from a particular chromosomal region have any role in determining its position in the nucleus? An elegant study using new tools that allow the positional mapping of all chromosomes now reveals insights into the rules that shape the three-dimensional arrangements of chromosomes and shows that generich regions are closer to the centre of the nucleus in human cells.

Recent technical advances have provided detailed information about the compartmentalization of chromosomes into discrete territories and their dynamic interactions with other nuclear components in different cell types. But how can we elucidate the rules that generate cell-type-specific, functionally relevant, higher-order chromatin arrangements? Bolzer et al. developed tools that helped them to overcome some of the technical limitations encountered by previous studies and explore some of their discrepancies. Differential colouring of all 24 chromosome types (22 autosomes plus $\mathrm{X}$ and $\mathrm{Y}$ ) in diploid male human fibroblasts was achieved by fluorescence in situ hybridization (FISH) using combinatorial labelling of chromosome paint probes and conditions that preserved the threedimensional nuclear shape.

Using the computer program
goldFISH to analyse information from 54 nuclei, the authors determined the three-dimensional locations of the fluorescence intensity gravity centres (IGCs) of individual chromosome territories (CTs) and of the nucleus. Their results show a probabilistic three-dimensional order of prometaphase chromosomes and CTs in nuclei of quiescent and cycling human fibroblasts. They also showed a non-random correlation with chromosome size: small chromosomes were preferentially located towards the centre of the nucleus, whereas large chromosomes were distributed closer to the nuclear rim. These findings in cells with flat, ellipsoidal nuclei also contradict previous studies in cells with rounder nuclei that showed preferential positioning of gene-rich CTs towards the centre of the nucleus.

Despite these differences between cell types, however, the authors did find evidence for a common rule that relates gene expression to nuclear position. Human chromosome 19 is gene-rich, whereas chromosome 18 is gene-poor. In spherical lymphocyte nuclei, chromosome 18 CTs are located at the nuclear periphery, whereas chromosome 19 CTs are positioned close to the nuclear centre. By contrast, in fibroblast nuclei, CTs from both chromosomes are distributed around the nuclear centre; however, whereas chromosome 18 CTs remained attached to the nuclear envelope, chromosome $19 \mathrm{CTs}$ were not. This finding is consistent with previous reports of an evolutionarily conserved 'shell' of gene-poor chromatin that covers the nuclear envelope.

A complex picture is emerging in which small chromosomes and gene-rich regions are located closer to the middle of the nucleus; whether this observation reflects an increased stability associated with this organization or whether it imparts protection of the centrally localized euchromatin against DNA damage remains to be elucidated. The results presented here shed light on the higher-order organization of nuclear architecture and inspired the authors to put forward questions for future experiments: do shape changes enforce changes in CT arrangements, or vice versa? And are changes of nuclear shape causally connected with changes in gene-expression patterns?

Ekat Kritikou

(2) References and links ORIGINAL RESEARCH PAPER Bolzer, A. et al. Three-dimensional maps of all chromosomes in human male fibroblast nuclei and prometaphase rosettes. PLoS Biol. 3, e157 (2005)

FURTHER READING Cremer, T. \& Cremer, C. Chromosome territories, nuclear architecture and gene regulation in mammalian cells. Nature $R e v$. Genet. 2, 292-301 (2001)

WEB SITE

Thomas Cremer's laboratory: http://www. biologie.uni-muenchen.de/ou/humbio/main_en/ solovei/topology.html 